\title{
Prospectiva estratégica: instrumento para a construção do futuro e para a elaboração de políticas públicas
}

\section{Introdução}

Este artigo pressupõe um Estado formulador de políticas públicas e detentor de instrumentos de planejamento, o que nos remete a um viés ideológico: de que, no capitalismo contemporâneo, Estado e mercado coordenem os sistemas econômicos, conforme explicitado no Plano Diretor da Reforma do Aparelho de Estado, de 1995. Só a aceitação dessa hipótese pode supor a existência e a necessidade de políticas públicas, remetendonos ao campo da ideologia e contrapondo-nos ao liberalismo.

Como quesito básico para a elaboração e a implementação de políticas públicas, coloca-se a existência de aparelho de Estado forte, com governança - considerada aqui como a capacidade técnica de implementar políticas públicas - e, claro, governabilidade. A fragilidade do aparelho do Estado brasileiro, privatizado há cinco séculos por grupos de interesses e por corporações, compromete significativamente o seu núcleo estratégico, lócus da formulação de políticas públicas.

A elaboração de políticas públicas pressupõe extenso ferramental de análise histórica, que nos possibilite quantificar e compreender a lógica dos diversos processos que interagem de forma positiva ou negativa com os sistemas, objetos dessas políticas. No entanto, o conhecimento do passado, o diagnóstico do presente e o desenho minucioso desses sistemas não são suficientes para a elaboração de políticas públicas que condicionem e orientem o futuro.

O voluntarismo, ou aquilo que, no senso comum, é chamado de "vontade política", não determina ou garante que um desejo de futuro seja
Carlos Manuel

Pedroso Neves

Cristo é

arquiteto pela

Universidade de

São Paulo

(USP) e cursou

o programa

Science et

Programation

Urbaine et

Régionale da

Universidade

Católica de

Louvain/

Bélgica.

Atualmente é

chefe de

gabinete da

Secretaria de

Tecnologia

Industrial do

Ministério do

Desenvolvimento,

Indústria e

Comércio

Exterior.

Contato:

carlos.cristo@

desenvolvimento. gov.br 
concretizado, nem sequer leva em conta alterações dos ambientes institucionais e organizacionais ou, então, mudanças tecnológicas possíveis de ocorre, que alterem profundamente tendências conhecidas.

Um exemplo fácil é o comportamento dos indicadores de qualidade ambiental das grandes metrópoles: eles tendem a uma correlação positiva, linear ou exponencial, com o aumento populacional ou da atividade econômica. A introdução de dispositivos legais (ambiente institucional), proibindo o uso de determinados combustíveis, como a lenha e o carvão em Londres, a obrigatoriedade do uso de catalisadores (tecnologia) em veículos ou ainda o surgimento de motores a hidrogênio rompem essas tendências e desenham novo futuro.

Karl Marx, no 18 Brumário de Luís Bonaparte, escreveu que "o homem constrói o seu futuro, mas não o faz da forma que gostaria", aludindo ao precário controle que a humanidade tem sobre o seu futuro. Esse texto é original na discussão e na crítica ao determinismo histórico.

A prospectiva estratégica aparece como ferramenta que, apoiada nos instrumentos de análise econômica e social existentes e em técnicas específicas, como o Delphi, a construção de cenários, a matriz de impactos cruzados e outras, possibilita “visões de futuro", que permitirão a elaboração de políticas públicas que visem à construção de futuro desejável.

Devo salientar que somente o conhecimento e a análise do passado e do presente, de forma profunda e consistente, possibilitarão a realização de estudos prospectivos ou de futuro. Segundo Michel Godet, "os modelos são invenções do espírito para representar um mundo que nunca se deixará encerrar na jaula das equações... Não há oposição, mas complementaridade entre intuição e razão. Para permanecer uma indisciplina intelectual, fecunda e credível, a prospectiva tem necessidade de rigor".

\section{História e conceitos gerais}

Se partimos do princípio de que o futuro não existe - ele ainda acontecerá -, qualquer previsão desse futuro deve ser entendida como exercício de charlatanismo ou de alto grau de incerteza.

Até um dos métodos mais comuns de previsão do futuro - a astrologia - embora não científico, não pretende, segundo "especialistas", ser determinista, mas apontar tendências, de acordo com "cenários" construídos a partir de mapas astrais, em que a conjugação dos astros é associada a determinados fenômenos, que induzirão o futuro dos indivíduos.

A prospectiva também não pretende ser determinista, mas possibilitar a redução de incertezas quanto a períodos futuros, baseando-se no conhecimento que as pessoas têm de determinados sistemas que serão "prospectados": ela precisa desvelar o provável comportamento desses atores 
na construção do futuro e antecipá-lo. Talvez o mais importante de um exercício de prospectiva seja o processo, e não o resultado final. É nele que se buscarão consensos sobre o futuro a ser realizado ou construído.

Os primeiros "construtores de futuro" de que temos conhecimento foram os profetas, presentes nas religiões judaica, cristã e islâmica. Ficanos claro que esses importantes personagens da história tiveram papel preponderante de coach, não pretendendo ser meros adivinhos. Na Grécia - o futuro era "predito" nos oráculos - o jogo de Delphos, ou método Delphi, homenageia o oráculo de Delphos. A preocupação com o futuro ressurge com o Renascimento e toma importante forma literária com Júlio Verne, no século XIX. No início do século XX, novos pensadores abordam o futuro: George Wells, Vernon Lee e Berthand Russel. Na década de 30, uma obra literária ficcionista torna-se famosa: $O$ admirável mundo novo, de Aldous Huxley. A obra de Verne inspira e direciona o futuro, que reproduz a sua arte. No caso de Huxley, a tecnologia caminha na direção apontada no livro e o alerta sobre a questão política foi, seguramente, importante instrumento para guiar o futuro numa direção melhor.

As duas guerras do século passado e sua herança assustadora, a guerra fria, obrigaram-nos ao desenvolvimento de instrumentos de planejamento que ultrapassassem os tradicionais planos qüinqüenais, fossem menos determinísticos e mais probabilísticos e buscassem evitar situações de catástrofe das quais poderíamos não ter retorno, dada a alta capacidade de autodestruição adquirida pela humanidade.

Nesse contexto, surgem, na década de 1960, a Rand Corporation, na Califórnia, e posteriormente o Hudson Institute, como centros de referência de estudos prospectivos. Notabilizaram-se figuras como Herman Kahn e Michel Godet, trabalhos como o World dynamic, sobre sistema ecológico, e o estudo prospectivo da Shell, que, em 1969, possibilitou uma visão futurística de possível choque do petróleo cuja conseqüente estratégia levou a Shell a obter petróleo em águas do Mar do Norte antes das demais concorrentes, o que a alçou ao segundo lugar no ranking da sua categoria.

Outros fatores pressionaram o desenvolvimento de estudos prospectivos, como as restrições aos gastos governamentais decorrentes da crise econômica originada do choque do petróleo de 1973, que obrigaram os governos a maior eficiência e, portanto, a melhor planejamento.

Mais recentemente, as profundas mudanças decorrentes da nova economia, com a aceleração da competitividade mundial e a alteração das geografias política e econômica, da valorização da ciência e tecnologia, as mudanças no processo produtivo industrial, com o surgimento das alianças estratégicas, redes e novas relações cliente-fornecedor, com o aumento do grau de complexidade nas atividades humanas, associado às novas infra-estruturas de comunicação e de processamento de dados, obrigam a uma vigília permanente em direção ao futuro. 
O estudo prospectivo australiano aponta como key forces for change:

- a integração global,

- a aplicação da informação e das tecnologias de comunicação,

- a sustentabilidade do meio ambiente,

- os avanços em tecnologias biológicas.

Em outras épocas, o pretexto para as mudanças foram as especiarias, "o caminho para as Índias", para os quais os exercícios de prospectiva consistiam em subir no mastro principal da caravela e olhar mais longe. Houve momentos em que o olhar para o futuro foi desprezado e tivemos desastres como o do Titanic.

Hoje não subimos em avião que não disponha de elementar ferramenta de prospectiva: o radar, em permanente funcionamento durante todo o vôo. No entanto, inúmeras organizações em muitos países acreditam que podem caminhar sem olhar para a frente ou de olhos tapados, rumo ao desastre.

Os ciclos evolutivos lentos, com mudanças tecnológicas distantes cronologicamente, possibilitavam previsão do futuro baseada em tendência passada, durante longos períodos de tempo. As tecnologias de informação e comunicação tornam esses ciclos muito curtos e a previsão do futuro torna-se exercício de muita incerteza. É lidar com essa incerteza e reduzi-la, antecipando os processos de ruptura ou de inovação, o objetivo principal dos estudos de prospectiva.

\section{Definições}

Ao se falar de prospectiva, encontramos, de forma associada, outros termos, como prognóstico e cenário:

- Prognóstico é usualmente entendido como a previsão de algo que vai acontecer. Os prognósticos meteorológicos, por exemplo, fazem parte do nosso dia-a-dia.

- Cenário é uma situação que possa apresentar-se como resultado de ação ou dinâmica evolutiva no tempo.

- Prospectiva é uma visualização do futuro, quando este não pode ser visto como simples extrapolação do passado, mas como conjunto de futuros possíveis, cada um em um cenário diferente.

Podemos prognosticar o tempo para os próximos dias, a inflação do ano, o resultado das próximas eleições e também fazer exercícios de prospectiva para identificar as mudanças climáticas da próxima década, do sistema político na China, das diferentes tecnologias no setor energético ou do tratamento de doenças auto-imunes.

Quando se relaciona prospectiva com planejamento e estratégia, podemos partir da definição de planejamento de Russel Ackoff (1973): 
"planejamento é conceber um futuro desejado, bem como os meios para lá chegar".

Como já foi mencionado, a importância maior da prospectiva é o processo, pois "o futuro começa hoje e somos atores chaves para a construção do futuro desejado" (Arapé, 2001). Nesse sentido, a prospectiva é o insumo básico do planejamento estratégico. Mediante a construção de cenários exploratórios, são identificados os desafios do futuro e, após um processo de decisão estratégica, obtidos os cenários prescriptores, que indicarão o futuro desejado.

Embora Michel Godet considere "a prospectiva e a estratégia dois amantes intimamente ligados", trata-se, na verdade, de duas entidades distintas. Por isso convém separar bem:

- tempo da antecipação, ou seja, a prospectiva das mudanças possíveis e desejáveis;

- tempo da preparação da ação, ou seja, a elaboração e avaliação das opções estratégicas possíveis para a organização se preparar para as mudanças esperadas (pré-atividade) e provocar as mudanças desejáveis (proatividade).

Esse mesmo autor apresenta, em função dessa dicotomia, quatro questões básicas:

- Que pode acontecer no futuro?

- Que posso fazer?

- Que vou fazer?

- Como vou fazê-lo?

E uma questão prévia e essencial:

- Quem sou eu?

É claro que a prospectiva dá conta da primeira questão; os tomadores de decisão, da segunda; e o processo de planejamento estratégico, das duas seguintes. A resposta à quinta questão, prévia e essencial, deve ser objeto de todos os atores envolvidos nos processos.

Talvez seja esta a questão mais difícil, dependendo do significado que lhe for atribuído. Michel Crozier afirma que "o problema é o problema", referindo-se à dificuldade maior em definir exatamente o que queremos ou o que somos.

Retornando a Godet, quatro atitudes são por ele descritas como possíveis diante do futuro:

- avestruz, que sofre a mudança;

- bombeiro, que, percebendo o fogo (a mudança), o combate;

- segurador, que se previne para a mudança;

- conspirador, que provoca a mudança.

Receio que falte outra categoria, associada à avestruz: os sonhadores. Os meios acadêmicos, comumente identificados como conspiradores, 
situam-se freqüentemente mais na esfera do desejo do que na da realidade, e os protagonistas da história acabam tendo outras origens.

É importante afirmar, como objetivo maior da prospectiva estratégica a identificação de cenários plausíveis, fruto de tendências dominantes e de cenários desejáveis, para que, por meio de políticas públicas, estreite-se a brecha entre essas duas situações e se construa um futuro desejável.

\section{O método}

Não é sem lógica que dispomos mais de análises do que de prognósticos. A análise é realizada ex post e, evidentemente, não trabalha com incertezas. Já o prognóstico depara-se com sistemas não estruturados, complexos, dinâmicos, ambíguos e incertos. Ausência de dados e fragilidades metodológicas e conceituais contribuem para dificultar a elaboração de prognósticos.

Podemos, por exemplo, citar a derrubada do muro de Berlim e a derrocada da União Soviética, acontecimentos tidos como inesperados até pelos melhores serviços de inteligência. Num patamar estratégico inferior, a situação econômica da Argentina era considerada, no início de 2000, promissora. Em 2002, a Argentina é vista como à beira de um abismo.

Como primeiro passo para um exercício de prospectiva, devemos responder à pergunta: “Quem somos?”, colocando-nos no lugar do objeto do estudo. O passo seguinte deve ser a perfeita definição do sistema observado. É extremamente importante a percepção sistêmica do objeto do estudo, considerando seus ambientes institucionais e organizacionais.

Embora, como nos referimos anteriormente, a modelização do futuro a partir de dados quantitativos do presente seja inócua, dadas as enormes incertezas quanto ao comportamento das variáveis que determinarão esse futuro, o conhecimento profundo da realidade e dessas variáveis é fundamental para a construção de cenários.

As técnicas mais comuns utilizadas em estudos prospectivos são:

- o modelo bayesiano;

- as matrizes de impacto cruzado;

- o Delphi;

- a brainstorming.

Dessas iremos nos deter mais no Delphi, técnica de painel de experts utilizada universalmente. As demais técnicas são freqüentemente utilizadas subsidiariamente para a construção de cenários.

Da apostila publicada pela Organização das Nações Unidas para o Desenvolvimento Industrial (Onudi), em junho de 2001, e elaborada por Jesús E. Arapé, diretor da Vision Grupo de Consultores da Venezuela, extraímos o seguinte sobre o Delphi: 
"Esta técnica tem por objeto (no contexto dos exercícios de prognóstico ou de prospectiva) a construção de cenários com relação ao comportamento de uma ou várias variáveis tomadas em consideração. Quando a ou as variáveis em consideração não podem ser medidas diretamente senão através de uma gama de indicadores associados, então a técnica deverá centrar-se no comportamento de tais indicadores para dali concluir acerca do comportamento de suas variáveis respectivas."

O Delphi, ou jogo de Delphos, baseia-se na convergência de opiniões sobre uma mesma questão. Essa convergência obtém-se mediante sucessivas "rodadas" de questionários a especialistas, sendo que a cada "rodada" é apresentada a tabulação da primeira e dada a opção de ser revista a opinião anterior. Normalmente em três "rodadas" é obtida a convergência.

Essa técnica pressupõe a identificação dos especialistas, pessoas com alto grau de conhecimento sobre as questões formuladas, independentemente de maior ou menor poder político nas organizações em que trabalhem. Não há preocupação maior com o número de especialistas envolvidos, dado que o exercício não é quantitativo, mas qualitativo, portanto não estatístico. Assim mesmo, considerando a dificuldade em se obterem respostas e as desistências no decorrer do processo, um número de participantes inferior a cem não é aconselhado.

Outra característica do método é a consulta individual e anônima, visando impossibilitar que haja influência de um especialista sobre o outro, ou que os extrovertidos "roubem a cena" aos demais, situação comum nas dinâmicas coletivas.

Posteriormente ao Delphi, surge o Método de Convergência de Opiniões. A diferença da técnica Delphi para a técnica de Convergência de Opiniões é que esta segunda - desenvolvida e operacionalizada por Bilich, no Instituto de Pesquisas Espaciais (INPE), a partir da primeira -, atribui pesos diferenciados às opiniões dos diferentes participantes das pesquisas, uma vez que suas qualificações para opinar sobre o problema em questão são diferenciadas. $\mathrm{O}$ valor/peso da opinião de cada participante é definido a partir de pesquisa com os participantes do estudo, que atribuem peso à sua própria importância e à importância dos demais participantes. De acordo com Bilich (apud Chaves, 1996, p. 28), "a técnica de Convergência de Opiniões faz parte do conjunto de técnicas que viabilizam previsões baseadas em julgamento. Esta técnica está também associada à do Painel de Especialistas e "se utiliza das opiniões de um grupo de especialistas em determinado assunto, articulando de maneira lógica, imparcial e sistemática essas opiniões, através de feedbacks controlados". 
O desafio maior desse ou de qualquer outro método utilizado para prognosticar o futuro continua sendo a precisa identificação e descrição do objeto do estudo. O rigor dessa identificação possibilitará formulação adequada do questionário Delphi.

A delimitação do objeto do estudo é feita normalmente por meio de um painel de stakeholders, podendo ser utilizada a técnica brainstorming, sobre a qual nos debruçaremos adiante. Delimitado o objeto de estudo, a sua descrição e o diagnóstico do sistema constituem os passos seguintes, fundamentais para a identificação dos fatores críticos. Para esse ponto do trabalho convergem as análises do contexto político (jogo dos atores, ambientes institucional e organizacional), da economia dos processos (eficiência) e das técnicas específicas de cada caso.

Os fatores críticos podem ser considerados como aqueles que impedem a convergência entre o futuro plausível e o desejável, identificados pelo desempenho das variáveis consideradas e respectivos indicadores. São esses fatores críticos que deverão ser estudados, e ter identificadas as suas variáveis e seus indicadores, possibilitando a elaboração do questionário Delphi. É importante retomar o conceito do estudo prospectivo para possibilitar "visão de futuro" das rupturas tecnológicas ou políticas que modifiquem tendências identificadas, dando outra direção ao futuro.

A técnica brainstorming consiste na reunião de um grupo, não superior a 12 pessoas, em volta de uma mesa, para a obtenção de alternativas à solução de determinado problema, exposto de forma clara ao grupo. É estimulada a vazão livre de idéias, preferencialmente incomuns, e é proibida a crítica. $\mathrm{O}$ exercício tem tempo limitado, definido a priori. Existem ainda técnicas para a organização e a consolidação dos resultados, como a espinha de peixe, oriunda do TQM. Uma nova versão para o brainstorming é o painel eletrônico, que possibilita a reunião de número maior de pessoas e tem a vantagem da não-identificação do autor de cada idéia. Para a realização desse painel, são necessários um microcomputador para cada participante e um para o monitor dos trabalhos.

A técnica Matrizes de Impactos Cruzados consiste em fazer uma exploração do futuro com base em uma série de eventos que podem ou não ocorrer num horizonte temporal considerado. É considerada como evento uma hipótese que pode ou não ser certa, conforme tal evento ocorra ou não no marco temporal analisado.

Nessa técnica, não basta identificar um conjunto de eventos cujas probabilidades de ocorrência especifiquem os cenários futuros, mas é necessário estabelecer as inter-relações das ocorrências entre uns e outros, isto é, o impacto cruzado nas ocorrências deles.

$\mathrm{Na}$ utilização dessa técnica, contamos hoje com softwares que facilitam extraordinariamente a sua utilização. 
O modelo bayesiano é também utilizado na prospectiva. Foi Bayes quem trouxe para a estatística a questão qualitativa, por meio do conceito da inferência. O exemplo clássico é a decisão de um juiz de indiciar ou não um suspeito por um crime, em função dos indícios de culpa existentes.

\section{Experiências internacionais}

\section{Áustria, 1998}

A Áustria optou por não direcionar o seu programa de prospectiva para a identificação de tendências gerais. Diferenciando as estratégias entre países grandes e pequenos no tocante a estudos prospectivos, esse país direcionou o seu trabalho para a identificação de esferas e nichos em que acredita poder alcançar posição de liderança nos próximos 15 anos.

Um grupo de especialistas levantou 40 hipóteses de inovação em sete áreas em que foram consideradas vantagens competitivas:

- novas formas de habitação e construção orientadas ao meio ambiente;

- tecnologia médica e de apoio a pessoas de idade;

- aprendizagem durante toda a vida;

- produção limpa e sustentável;

- alimentos orgânicos;

- mobilidade física;

- materiais.

Realizada a consulta Delphi, o estudo aponta, para a Áustria, oportunidades especiais em:

- modelos de simulação em processos de construção;

- materiais de baixo peso e alta tecnologia;

- reciclagem de materiais compostos e materiais mistos;

- equipamento de redução de ruído para ferrovias;

- tecnologias de produção mais limpa (especialmente em metais e papel);

- madeira como material para construção;

- construção ecologicamente racional;

- alimentos orgânicos;

- sucedâneos de órgãos e funções;

- tecnologias de informação e comunicação (nichos específicos).

O estudo austríaco foi amplamente divulgado, sobretudo nos meios acadêmicos e científicos, e produziu impacto nas políticas de pesquisa e desenvolvimento, além de ter fomentado a multidisciplinaridade, a cooperação e a formação de redes. 
A União Européia apresentou em Bruxelas, em fevereiro de 2000, o resultado do trabalho conduzido pelo Instituto para Estudos em Prospectiva Tecnológica (IPTS), um dos "Joint Research Institutes" da UE, sediado em Sevilha.

O estudo denominado "Futures Project" consumiu dois anos de trabalho e constitui-se no mais importante estudo regional de prospectiva jamais realizado. Todos os seus resultados estão disponíveis na página da WEB http://futures.jrc.es para download.

O objetivo do trabalho foi examinar os efeitos individuais e combinados dos drivers tecnológicos, econômicos, políticos e sociais no período de 2000 a 2010. Houve uma preocupação central com tecnologia, competitividade e emprego.

Os temas principais abordados foram:

- "dínamo" tecnológico (foi produzido um "mapa tecnológico", indicando debilidades, fortalezas e targets europeus);

- a Europa na sociedade global do conhecimento;

- a sociedade européia conduzindo a mudança (alguns dos pontos destacados foram a seguridade social, a educação, a saúde e o meio ambiente).

Foram utilizados painéis de especialistas, com brainstorming eletrônico, workshops, e todo o trabalho foi alicerçado em papers de pesquisa.

Os painéis centraram-se nos seguintes key drivers:

- contexto político e econômico;

- rumo social e demográfico;

- recursos naturais e meio ambiente;

- tecnologia da informação e da comunicação e sociedade da informação;

- ciências da vida e fronteiras da vida.

O passo seguinte, por meio de workshops, foi integrar esses resultados com três áreas chaves para a formulação de políticas públicas: tecnologia, emprego e competitividade. Adicionalmente foram cruzados outros três temas: ensino e conhecimento, alargamento da UE e custo social. Essa fase exigiu extenso trabalho de pesquisa, workshops de consolidação e um seminário final, antes da elaboração do relatório conclusivo, apresentado em fevereiro de 2000.

\section{Nova Zelândia, julho de 1999}

A Nova Zelândia caracteriza-se por ser um país pequeno, isolado, porém com elevada renda per capita e excelentes indicadores sociais, que refletem igualmente excelente distribuição da riqueza. $O$ sucesso desse 
país deve-se à capacidade de formular e implementar políticas públicas, que lhe confere alto grau de competitividade.

Em julho de 1997, iniciou-se nesse país um projeto de prospectiva tecnológica, visando:

- a um processo estratégico envolvendo diversos setores, grupos e comunidades, com especial atenção às necessidades e oportunidades futuras em ciência e tecnologia;

- ao desenvolvimento de novo conjunto de prioridades para investimentos governamentais em pesquisa, ciência e tecnologia.

As fases de desenvolvimento desse projeto foram:

- julho/97 a março/98 - estabelecimento de contexto para pensar sobre as necessidades de conhecimento futuro;

- março/98 a outubro/98 - desenvolvimento de estratégias setoriais;

- agosto/98 a março/99 - tomada de decisão do governo sobre novas prioridades e estratégias de investimento;

- a partir de julho/99 - início da implementação das novas prioridades e estratégias de investimento.

É interessante ressaltar o que a Nova Zelândia considera como elementos chaves para cenários úteis: reunir informação, construir cenários e pesquisar o futuro.

Quanto ao meio ambiente, os temas resultantes do estudo de maior impacto nas próximas duas décadas são:

- biodiversidade;

- recursos hídricos;

- energia;

- dejetos;

- mudanças climáticas;

- deterioração da camada de ozônio.

\section{Austrália, maio de 1996}

Uma importante contribuição do estudo prospectivo autraliano foi o envolvimento dos stakeholders na reflexão sobre o futuro e sobre as suas próprias demandas sociais, políticas, econômicas e tecnológicas. O Conselho Australiano de Ciência e Tecnologia (Astec), que contratou o estudo, explicitou com clareza a importância do processo como ferramenta de mudança de atitude e de comprometimento da indústria com a pesquisa e o desenvolvimento.

Ressaltou-se a importância da interdisciplinaridade e da integração entre ciência, tecnologia e economia. Assumiu-se o pequeno papel da Austrália como produtora mundial de C\&T, por isso mesmo de difícil planejamento, e de muita importância. 
Na primeira fase do estudo prospectivo, foram identificadas as "forças-chave para mudança para 2010":

- integração global;

- tecnologias de informação e comunicação aplicadas;

- meio ambiente sustentável;

- avanços em biotecnologia.

As ações estratégicas, para enfrentar o desafio imposto pelas "forças-chave para mudança para 2010", foram assim definidas:

- desenvolver um conjunto de princípios estratégicos para guiar a Austrália a participar nos vários fóruns internacionais e encorajar e promover o benchmarking nas empresas autralianas;

- assegurar que as tecnologias de informação e comunicação estão integradas como componente chave dentro de todos os setores e que os australianos estão habilitados a utilizá-las;

- estabelecer parâmetros para o sistema de ressource accounting, necessidades para as áreas científica e tecnológica e o desenvolvimento de infraestrutura e pesquisa estratégica nessas áreas;

- desenvolver diretrizes para questões éticas, ambientais e de equidade surgidas a partir do desenvolvimento da biotecnologia.

\section{O "estado da arte" no Brasil e conclusões}

Um pioneiro na conceituação de estudos do futuro foi o professor Henrique Rattner, da Fundação Getúlio Vargas, de São Paulo, ao publicar, em 1979, Estudos do futuro: introdução à antecipação tecnológica e social. Ainda em São Paulo desenvolveu-se o Laboratório de Estudos do Futuro da Universidade de São Paulo. Instituições como o $\mathrm{CPqD}$ da Telebrás, em Campinas, a Petrobras, a Eletronorte, a Embraer, o Banco do Brasil, a Embrapa, entre outras, adotam a prospectiva como ferramenta fundamental no seu direcionamento tecnológico. Universidades, como a Universidade do Brasil e a Unicamp, vêm desenvolvendo atividades de prospectiva tecnológica.

No governo federal, dois ministérios iniciam programas de prospectiva: o de Ciência e Tecnologia, que desenvolve, a partir de 2000, o Prospectar, consultando, na primeira rodada do seu Delphi, 40 mil especialistas de diversas áreas da ciência e tecnologia com o objetivo de direcionar a alocação dos recursos dos fundos setoriais. No Ministério do Desenvolvimento, Indústria e Comércio Exterior, por meio de sua Secretaria de Tecnologia Industrial e com o apoio da Organização das Nações Unidas para o Desenvolvimento Industrial (Onudi), desenvolve-se, também a partir de 2000, o Programa Brasileiro de Prospectiva Tecnológica Industrial, no contexto de uma iniciativa da Onudi denominada Prospectiva Tecnológica 
para a América Latina e Caribe, envolvendo os países da região. O programa brasileiro, desenvolvido em parceria com o Instituto de Pesquisas Tecnológicas de São Paulo, a Escola Politécnica da Universidade de São Paulo, a Escola de Química da Universidade do Brasil (Universidade Federal do Rio de Janeiro) e o Senai/Cetiqt, abordando as cadeias produtivas de madeira e móveis, construção civil, transformados plásticos, têxteis e confecções, é referência para os demais países da região.

Apesar dessas iniciativas, a prospectiva estratégica ou tecnológica - dependendo do foco - ainda é desconhecida da grande maioria das organizações e foi apontada, por recente estudo sobre oferta e demanda de tecnologia, patrocinado pela Confederação Nacional da Indústria (CNI), como o instrumento mais ausente no âmbito da questão tecnológica.

A construção de futuro desejável é possível, e, embora ele não resulte de desígnio tecnocrático, a correta e a eficiente utilização de instrumentos adequados, como a prospectiva, poderá contribuir para o êxito de sua construção.

\section{Referências bibliográficas}

AcKoff, Russel. Méthodes de planification d'entreprise. Paris: Les Éditions d'Organisation, 1973.

ArAPÉ, Jesús E. Programa de Prospectiva Tecnológica para Latinoamérica y Caribe. Manual de Metodologias, Tomos I, II, III y IV. Viena: Onudi, 2001.

Astec. Futures Needs 2000. www.dist.gov.au/science/astec/astec/future/final/futurea.html Bresser Pereira, Luiz Carlos. Reforma do Estado para a cidadania. São Paulo: Editora 34, 1998.

CAstro, Antônio Maria et al. Cadeias produtivas e sistemas naturais: prospecção tecnológica. Brasília: Embrapa, 1998.

Chaves, Fernando Antonio Vieira. Análise da cultura organizacional e administração estratégica utilizando a técnica de convergência de opiniões. Dissertação de Mestrado. Universidade de Brasília, Faculdade de Estudos Sociais Aplicados, Departamento de Administração, maio de 1996.

ERKER, Erfried. Reporte Delfos de Áustria. Viena: Onudi, 2000.

Godet, Michel. A caixa de ferramentas da prospectiva estratégica. Lisboa, 2000.

. Crise de la prévision essor de la prospective. Paris: PUF, 1977.

IPTS. The IPTS Futures Project Synthesis Report. Sevilha, 2000.

Johson, Ron. A importância de olhar no futuro. Revista Inteligência Empresarial, n ${ }^{\circ} 5$. Coppe, UFRJ, Rio de Janeiro, 2000. 
Marcial, Elaine Coutinho. Aplicação de metodologia de cenários no Banco do Brasil no contexto da inteligência competitiva. Dissertação de mestrado. Université de Droit et dês Sciences D’Aix-Marseille. Marseille, 1999.

MDIC. Plano de Ação do Programa Brasileiro de Prospectiva Tecnológica Industrial. Brasília, 2000.

RATTNER, Henrique. Estudos do futuro: introdução à antecipação tecnológica e social. São Paulo: Editora da Fundação Getulio Vargas, 1979. 


\section{Prospectiva estratégica: instrumento para a construção do futuro e para a elaboração de políticas públicas}

Carlos Manuel Pedroso Neves Cristo

A prospectiva estratégica aparece como ferramenta que, apoiada nos instrumentos de análise econômica e social existentes e em técnicas específicas, como o Delphi, a construção de cenários, a matriz de impactos cruzados e outras, possibilita "visões de futuro", que permitirão a elaboração de políticas públicas que visem à construção de futuro desejável.

Este trabalho pressupõe um Estado formulador de políticas públicas e detentor de instrumentos de planejamento, o que nos remete a um viés ideológico: de que, no capitalismo contemporâneo, Estado e mercado coordenem os sistemas econômicos, conforme explicitado no Plano Diretor da Reforma do Aparelho de Estado (Governo Federal, Brasil, 1995). Só a aceitação dessa hipótese pode supor a existência e a necessidade de políticas públicas, remetendo-nos ao campo da ideologia e contrapondo-nos ao liberalismo.

Após introdução ao tema, no primeiro capítulo, são apresentados, no segundo, os conceitos utilizados e um breve relato histórico dos processos de construção do futuro, desde os oráculos à atualidade. Já o terceiro capítulo apresenta as definições e os conceitos específicos utilizados neste trabalho, uma vez que prognóstico, cenário e prospectiva podem sugerir outros significados. Nesse capítulo é discutida a relação entre prospectiva e estratégia.

O quarto capítulo aborda a questão metodológica referente a essa ferramenta. São enunciadas as diversas técnicas utilizadas, com ênfase no questionário Delphi e no posterior Método de Convergência de Opiniões.

O penúltimo capítulo faz breve relato de algumas importantes experiências internacionais: Áustria, União Européia, Nova Zelândia e Austrália. Esse capítulo aborda ainda o estado da arte no Brasil e apresenta conclusões.

A metodologia adotada na elaboração deste trabalho foi a da revisão bibliográfica considerada mais significativa e a pesquisa, pela Internet, principalmente no que diz respeito às experiências internacionais.

\section{Prospectiva estratégica: instrumento para la construcción del futuro y para la elaboración de política pública}

\section{Carlos Manuel Pedroso Neves Cristo}

La prospectiva estratégica es la herramienta que permitirá la elaboración de las políticas públicas que tengan el objetivo de la construcción del futuro deseable. Ella está apoyada en los instrumentos existentes del análisis económico y social, en técnicas específicas, como el Delphi para la construcción de las escenas, de la matriz de impactos cruzados y de otras.

Este trabajo considera la existencia de un Estado capable de formular política y poseedor de los instrumentos de planeamiento, lo que conduz a nosotros por un viés ideológico: en el capitalismo contemporaneo el Estado se asocia al Mercado para la coordinación de los sistemas económicos, como explicitado en el "Plan de la Reforma del Estado" (Gobierno Federal, Brasil, 1995). Solamente por la aceptación de esta hipótesis es que se puede suponer la existencia y la necesidad de políticas públicas, remitiendo al campo de la ideología y oponiéndo al del liberalismo.

Después de la introducción del tema del primer capítulo, se presentam, en el segundo, los conceptos generales empleados y una sistesis histórica de los procesos de la construcción del futuro, desde los oráculos hasta hoy en día. El tercer capítulo presenta las definiciones
Revista do

Serviço

Público

Ano 54

Número 1

Jan-Mar 2003

Carlos Manuel

Pedroso Neves

Cristo é

arquiteto pela

Universidade de

São Paulo

(USP) e cursou

o programa

Science et

Programation

Urbaine et

Régionale da

Universidade

Católica de

Louvain/

Bélgica.

Atualmente é

chefe de

gabinete da

Secretaria de

Tecnologia

Industrial do

Ministério do

Desenvolvimento,

Indústria e

Comércio

Exterior.

Contato:

carlos.cristo@

desenvolvimento. gov.br 
y los conceptos específicos usados en este trabajo, de manera que el pronóstico, escena, prospectiva no puedan sugerir otros significados. En este capítulo se discute la relación entre la prospectiva y la estrategia.

El cuarto capítulo acerca la pregunta metodológica de esa herramienta. Son enumeradas as distintas técnicas usadas, con énfasis en el cuestionario de Delphi y el posterior Método de Convergencia de Opiniones.

El penúltimo capítulo hace un breve reporte de algunas experiencias internacionales importantes: Austria, Unión de Européia, Nueva Zelândia y Australia. Este capítulo, todavía, acerca el estado da arte en Brasil y presenta conclusiones.

La metodología adoptada en la elaboración de este trabajo consiste de la revisión bibliográfica y de la investigación, tras Internet, principalmente aquella que se refiere a las experiencias internacionales.

\section{Strategic prospective: instrument for the construction of the future and for the elaboration of public policies}

Carlos Manuel Pedroso Neves Cristo

Strategic prospective appears as a tool that supported by the existing instruments of economic and social analysis and in specific techniques such as Delphi, the construction of scenarios and the matrix of crossed impacts, among others, generates "visions of the future", that will allow for the elaboration of public policies that aim at the construction of a more desirable future.

This work presumes the State as an elaborator of public policies equipped with planning instruments, which sends to us an ideological bias: in the contemporary capitalism the State and market co-ordinate the economic systems, as shown in the Managing Plan of the Reformation of the State Device (Federal Government, Brazil, 1995). It is only by accepting this hypothesis that we assume the existence and the necessity of public policies, sending us back to the field of ideology and opposing liberalism.

After introducing the subject in the first chapter, the second chapter presents the general concepts used, together with a brief history of the processes of construction of the future, from the oracles to present time. Already the third chapter presents the definitions and specific concepts used in this work, in view that words such as prognostic, scene and prospective may suggest other meanings. That chapter also discusses the correlation between prospective and strategy.

The fourth chapter approaches the methodological question referring to this tool. A range of techniques used are enunciated, with emphasis in the Delphi questionnaire and the posterior Method of Convergence of Opinions.

The penultimate chapter gives a brief story of important international experiences: Austria, European Union, New Zealand and Australia. Still, this chapter presents the state of the art in Brazil together with the respective conclusions.

The methodology adopted in the elaboration of this work was the bibliographical revision, considered more significant, and also Internet research, mainly for the reference of the international experiences. 